\author{
Marquette University \\ e-Publications@Marquette
}

School of Dentistry Faculty Research and

Publications

Dentistry, School of

$12-2002$

\title{
NASA Light-Emitting Diodes for the Prevention of Oral Mucositis in Pediatric Bone Marrow Transplant Patients
}

\author{
Harry T. Whelan \\ Medical College of Wisconsin \\ James F. Connelly \\ Medical College of Wisconsin \\ Brian D. Hodgson \\ Marquette University, brian.hodgson@marquette.edu \\ Lori Barbeau \\ Children's Hospital of Wisconsin
}

A. Charles Post

Children's Hospital of Wisconsin

See next page for additional authors

Follow this and additional works at: https://epublications.marquette.edu/dentistry_fac

Part of the Dentistry Commons

\section{Recommended Citation}

Whelan, Harry T.; Connelly, James F.; Hodgson, Brian D.; Barbeau, Lori; Post, A. Charles; Bullard, George; Buchmann, Ellen V.; Kane, Mary; Whelan, Noel T.; Warwick, Ann; and Margolis, David, "NASA Light-Emitting Diodes for the Prevention of Oral Mucositis in Pediatric Bone Marrow Transplant Patients" (2002). School of Dentistry Faculty Research and Publications. 4.

https://epublications.marquette.edu/dentistry_fac/4 


\section{Authors}

Harry T. Whelan, James F. Connelly, Brian D. Hodgson, Lori Barbeau, A. Charles Post, George Bullard, Ellen V. Buchmann, Mary Kane, Noel T. Whelan, Ann Warwick, and David Margolis 


\title{
NASA Light-Emitting Diodes for the Prevention of Oral Mucositis in Pediatric Bone Marrow Transplant Patients
}

\author{
HARRY T. WHELAN, M.D., ${ }^{1}$ JAMES F. CONNELLY, M.D., ${ }^{1}$ BRIAN D. HODGSON, D.D.S., ${ }^{2}$ \\ LORI BARBEAU, D.D.S., ${ }^{2}$ A. CHARLES POST, D.D.S., ${ }^{2}$ GEORGE BULLARD, D.D.S., ${ }^{2}$ \\ ELLEN V. BUCHMANN, B.S., ${ }^{1}$ MARY KANE, B.S., ${ }^{1}$ NOEL T. WHELAN, B.S., ${ }^{1}$ \\ ANN WARWICK, M.D. ${ }^{3}$ and DAVID MARGOLIS, M.D. ${ }^{3}$
}

\begin{abstract}
Objective: The purpose of this study was to determine the effects of prophylactic near-infrared light therapy from light-emitting diodes (LEDs) in pediatric bone marrow transplant (BMT) recipients. Background Data: Oral mucositis (OM) is a frequent side effect of chemotherapy that leads to increased morbidity. Nearinfrared light has been shown to produce biostimulatory effects in tissues, and previous results using nearinfrared lasers have shown improvement in OM indices. However, LEDs may hold greater potential for clinical applications. Materials and Methods: We recruited 32 consecutive pediatric patients undergoing myeloablative therapy in preparation for BMT. Patients were examined by two of three pediatric dentists trained in assessing the Schubert oral mucositis index (OMI) for left and right buccal and lateral tongue mucosal surfaces, while the patients were asked to rate their current left and right mouth pain, left and right xerostomia, and throat pain. LED therapy consisted of daily treatment at a fluence of $4 \mathrm{~J} / \mathrm{cm}^{2}$ using a $670-\mathrm{nm}$ LED array held to the left extraoral epithelium starting on the day of transplant, with a concurrent sham treatment on the right. Patients were assessed before BMT and every 2-3 days through posttransplant day 14. Outcomes included the percentage of patients with ulcerative oral mucositis (UOM) compared to historical epidemiological controls, the comparison of left and right buccal pain to throat pain, and the comparison between sides of the buccal and lateral tongue OMI and buccal pain. Results: The incidence of UOM was $53 \%$, compared to an expected rate of $70-90 \%$. There was also a $48 \%$ and $39 \%$ reduction of treated left and right buccal pain, respectively, compared to untreated throat pain at about posttransplant day $7(p<0.05)$. There were no significant differences between sides in OMI or pain. Conclusion: Although more studies are needed, LED therapy appears useful in the prevention of OM in pediatric BMT patients.
\end{abstract}

\section{INTRODUCTION}

$\mathbf{O}$ RAL MUCOSITIS (OM) is a frequent side effect of chemotherapy in preparation for bone marrow transplant (BMT). Ulcerations in the vulnerable oral mucosa produce severe pain, oral superinfections that may lead to systemic infections, and compromise oral hydration and nutrition. ${ }^{1-3}$

Investigations into low-energy stimulation of tissues by lasers have shown increased cellular activity during wound healing, including increased collagen production ${ }^{4,5}$ and angiogenesis. ${ }^{6}$ The data suggest that monochromatic, near-infrared laser biostimulation produces its primary effect during the cell proliferation phase, ${ }^{7,8}$ increasing mitochondria respiration through stimulation of cytochrome oxidase.,5,9 Production increases have been seen in fibroblasts, collagen and procollagen, growth factors, lymphocytes, and extracellular matrix - as well as macrophage stimulation-with laser treatment. ${ }^{10-13}$ Optimal wavelengths for wound healing, as proven in previous laser studies, include 680, 730, and $880 \mathrm{~nm} \cdot{ }^{4,5,11,14}$ Additionally, previous studies have shown that use of helium-neon lasers in BMT recipients significantly decreases the severity and duration of ulcerative mucositis. ${ }^{15-17}$

Recently, light-emitting diodes (LEDs) have been shown to be a safe, efficient, lightweight, and less-expensive alternative 
to treat wounds. ${ }^{18}$ Originally developed for NASA plant growth experiments in space, LEDs can be designed to emit monochromatic light at wavelengths shown to be effective in laser light to treat wounds. ${ }^{19}$ LEDs have several advantages over lasers for clinical use. LEDs can be designed to emit a combination of wavelengths optimal for wound healing. They are compact, light, and require significantly less energy. Unlike lasers, they can be arranged in large, flat arrays allowing for the treatment of a wide, three-dimensional surface. The emitted near-infrared light has been shown to penetrate up to $23 \mathrm{~cm}$ of skin and muscle.,5,20 Because LED light is produced out-ofphase, it emits very little heat. Therefore, at intensities required to penetrate deeper tissues, there is little risk of heat damage to the treated epithelial tissues. Because of this nonsignificant risk (NSR) of heat damage to tissues, including the retina, the FDA has given NSR approval for therapeutic trials of LEDs in humans.

There is mounting evidence that LED arrays at the same biostimulatory wavelengths of previous laser studies have similar biochemical effects. Wong-Riley et al. have demonstrated LED stimulation of cytochrome c oxidase activity in tetrodotoxinpoisoned neurons. ${ }^{21}$ Earlier reports from our group have shown increased in vitro production of mouse-derived fibroblasts, ratderived osteoblasts, rat-derived skeletal muscle cells, and normal human epithelial cells with LED treatment. ${ }^{18,22-24}$

In our latest in vivo studies ${ }^{18}$ using an ischemic wound model in rats, we demonstrated decreased healing time of standardized wounds. Preliminary experiments on human wounds produced significant decreases in healing time in crewmembers aboard a U.S. Navy submarine and improvement in musculoskeletal training injuries in Navy SEAL team members with LED treatment. Lastly, preliminary data on pediatric BMT patients showed a $47 \%$ reduction in buccal pain of LEDtreated OM compared to the untreated throat.

Our purpose for this study is to determine objectively the severity and extent of mucositis in LED-treated buccal and lateral tongue mucosal surfaces compared to historical epidemiological data. We also set out to compare perceived mouth pain between the treated mouth and untreated throat as well as mouth xerostomia.

\section{MATERIALS AND METHODS}

We studied the effect of prophylactic LED treatments in pediatric patients undergoing chemotherapy in anticipation for BMT. Subjects were recruited consecutively among the patients being treated by the Bone Marrow Transplant Program at the Children's Hospital of Wisconsin in Milwaukee, Wisconsin from June 2000 to December 2001. The institutional review boards of Children's Hospital of Wisconsin and the Medical College of Wisconsin accepted and approved the study design.

Eligible participants were pediatric patients undergoing chemotherapy and/or radiation programs considered to be myeloablative in preparation for either autologous or allogenic BMT; patients with the emotional, cognitive, and mental maturity sufficient to tolerate light application and oral examination without combativeness; and patients whose parents or guardians were willing to give informed consent. Patients were excluded if they did not wish to submit to the trial protocol, were pregnant, had photophobia or were on medication that may cause epidermal or ocular photosensitivity, were younger than 2 years old, or had significant pulmonary dysfunction such that there was a risk of intubation during the first 21 days posttransplant.

\section{Study population}

Thirty-two patients were enrolled in the study (one patient received a second BMT 4 months later). The study population is summarized in Table 1. Mean age was 12.5 years, with a range of 3-23 years. Sixty percent of patients had a diagnosis of acute leukemia; other diagnoses included lymphoma, sarcoma of various types, chronic leukemia, severe aplastic anemia, myelodysplastic syndrome, neuroblastoma, and sickle cell anemia.

The myeloablative preconditioning for the patients in our sample is summarized in Table 2. Twenty-two patients $(66 \%)$ received total-body irradiation (TBI) in addition to chemotherapy. A majority of patients (53\%) received the combination cytarabine-cyclophosphamide-TBI.

Characterization of the preconditioning regimen is important in order to compare our results to historical controls. In the longitudinal study of OM by Woo et al., high rates of ulcerative oral mucositis (UOM) were seen in BMT patients undergoing cytarabine-cyclophosphamide-TBI (67\%); cyclophosphamideTBI (79\%); and melphalan (100\%). ${ }^{25}$ Similarly, Wingard, et al. found a high rate of UOM $(73 \%)$ in BMT patients undergoing busulfan-cyclophosphamide regimens. ${ }^{26}$ Finally, in Barasch et al., all 22 BMT patients had bilateral UOM despite singlesided helium-neon laser treatments. ${ }^{27}$ All but one patient had busulfan as part of their preparative regimen. From these data, it is within reason to expect a $70-90 \%$ rate of UOM in our patient population.

\section{Table 1. Patient Population}

\begin{tabular}{lc}
\hline Characteristic & $\begin{array}{c}\text { No. of } \\
\text { patients }\end{array}$ \\
\hline Male & $20(63 \%)$ \\
Female & $12(38 \%)$ \\
Age range (years) & $2-23$ \\
Mean age (years) & 12.5 \\
Diagnosis & \\
Leukemia & \\
$\quad$ Acute lymphocytic leukemia & $12(38 \%)$ \\
$\quad$ Acute myleogenous leukemia & $7(22 \%)$ \\
$\quad$ Chronic lymphocytic leukemia & $2(6 \%)$ \\
Lymphoma & \\
$\quad$ Hodgkin's lymphoma & $1(3 \%)$ \\
$\quad$ Non-Hodgkin's lymphoma & $2(6 \%)$ \\
Sarcoma & $3(9 \%)$ \\
Severe aplastic anemia & $2(6 \%)$ \\
Myelodysplastic syndrome & $1(3 \%)$ \\
Sickle cell anemia & $1(3 \%)$ \\
Neuroblastoma & $1(3 \%)$ \\
Total 32 (100\%) & \\
\hline &
\end{tabular}


TABle 2. BMT Preconditioning Regimens

\begin{tabular}{lc}
\hline Regimen & $\begin{array}{c}\text { No. of } \\
\text { patients }\end{array}$ \\
\hline Cytarabine-cyclophosphamide-TBI & $17(53 \%)$ \\
Etoposide-melphalan-TBI & $3(9 \%)$ \\
Melphalan & $3(9 \%)$ \\
Cytarabine-cyclophosphamide & $2(6 \%)$ \\
Busulfan-cyclophosphamide-TBI & $2(6 \%)$ \\
Busulfan-cyclophosphamide & $1(3 \%)$ \\
Busulfan-fludrabine & $1(3 \%)$ \\
Etoposide-carmustine-cytarabine & $1(3 \%)$ \\
Thiotepa-topotecan-carboplatin & $1(3 \%)$ \\
Cytarabine & $1(3 \%)$ \\
\hline
\end{tabular}

\section{Trial design}

Each patient enrolled in the study received a pre-BMT examination of their buccal and lateral tongue mucosa by two of three pediatric dentists trained in assessing the Schubert Oral Mucositis Index (OMI).28 Photographs of each side of the mouth were taken, and each observer independently rated the seven different categories for each side of the buccal and lateral tongue mucosal surfaces. Observers scored, on a 0-3 scale, categories that included atrophy, edema/cellulitis, erythema, hyperkeratosis, lichenoid formation, ulceration, and pseudomembrane formation. The ulceration category was scored according to the size $\left(1=<1 \mathrm{~mm}^{2}, 2=1-2 \mathrm{~mm}^{2}\right.$, and $3=>3 \mathrm{~mm}^{2}$ ). Patients were also given a self-reported pain and xerostomia (labeled "dryness") sheet containing the WongBaker "smiley face" scale ${ }^{29}$ for each set of the two symptoms. Participants were asked to rate their current level of oral pain located in the left cheek, the right cheek, and the throat. They were then asked to rate their current level of oral xerostomia in the left cheek and the right cheek.

After the patients received their BMT, they were examined again and were asked to rate their mouth and throat pain as well as their mouth xerostomia as described above. Patients were assessed every Monday, Wednesday, and Friday through posttransplant day 14 . We selected a stop date of 14 days in order to minimize the potential influence of mucosal changes due to graft-versus-host disease (GVHD) associated with allogenic BMT.

Patients then began LED exposure therapy on the first posttransplant day. LED treatment consisted of once per day exposure to light emitted from a $7.62 \mathrm{~cm} \times 12.7 \mathrm{~cm}$ array of LEDs (Quantum Devices, Barneveld, WI) at a wavelength of $670 \mathrm{~nm}$ with a homogeneous and consistent power exposure of $56 \mathrm{~mW} / \mathrm{cm}^{2}$. The array was held in close proximity (within $1 \mathrm{~cm}$ ) to the left extraoral epithelium of the cheek for a time calculated to achieve an energy level of $4 \mathrm{~J} / \mathrm{cm}^{2}$ (71 sec). All treatment sessions were conducted by trained clinicians familiar with the protocol for the placement of the LED array. Although no retinal damage from LEDs has been reported in the literature, we used protective eyewear, both to shield the patient's eyes and to blind patients during each treatment session. At the same treatment session, while the patient continued to wear the opaque protective goggles, the right side was treated for the same amount of time; however, an obstructing piece of foil was placed between the LED array and the patient. Both the patients and the observers were blinded as to which side was the actively treated side. Additionally, direct and scattered LED light would not be able to penetrate the tissues of the throat, therefore giving us an untreated control.

The outcomes of this study were the (1) percentage of patients with ulcerative mucositis compared to historical epidemiological controls, (2) the comparison of left and right buccal pain to throat pain, (3) the comparison of OMI between left and right buccal and left and right lateral tongue mucosal surfaces, and (4) interobserver consistency.

\section{Statistical analysis}

At each observation, the total of the seven OMI categories for each of the four sites were averaged between the two observers. At each observation posttransplant, the averaged total OMI score at each site was subtracted from the baseline total OMI average for each site. The same was done for the subjective pain and xerostomia scales.

To determine if differences between left and right sides in the objective and subjective scales existed, we used a singlesample, two-tailed Student's $t$ test of the differences of left and right buccal OMI, tongue OMI, buccal pain, and mouth xerostomia. To ascertain if significant differences existed between left and right buccal pain and throat pain, we used a paired, two-tailed Student's $t$ test. Interobserver variance was measured through a single-sample, two-tailed Student's $t$ test of the differences of each observation at each site between each observer pair.

The presence of UOM and its severity were determined by the maximum averaged ulceration score at any site during the study period. Maximum average ulceration scores in the range of 3.0-2.5 were counted as "severe;" ranges of 2.4-1.5 were counted as "moderate;" ranges of 1.4-0.5 were counted as "mild." The absence of UOM required having no note of ulceration among any observer at all sites through the 14-day time period.

\section{RESULTS}

Table 3 shows the results of the average difference of buccal and lateral tongue OMI, pain, and xerostomia scores from baseline at each observation number. There was no significance found between the left and right of buccal OMI, lateral tongue OMI, buccal xerostomia scale, or buccal pain scale at all observations. The measured OMIs are graphically represented in Figure 1. The buccal OMI peaks at observation 4, corresponding to approximately post-transplantday 9. Lateral tongue OMI peaks at observation 5, or posttransplant day 11-12. Because the buccal and tongue mucosal surfaces are so different, no comparison between them was made.

For the perceived pain data, there was significance $(p<0.05)$ between left buccal pain and throat pain as well as between right buccal pain and throat pain at observation 3 (Fig. 2). This 
Table 3. Calculated Average OMi, Pain, and Xerostomia Scores from Baseline

\begin{tabular}{lrcccccr}
\hline \multicolumn{1}{c}{$\begin{array}{c}\text { Observation number } \\
\text { Average posttransplant day }\end{array}$} & 1 & 2 & 3 & 4 & 5 & 6 & \\
\cline { 2 - 6 } & $1.8 \pm 0.2$ & $4.5 \pm 0.2$ & $6.7 \pm 0.1$ & $8.9 \pm 0.2$ & $11.5 \pm 0.2$ & $13.5 \pm 0.1$ & pvalue \\
\hline Left buccal OMI & $0.4 \pm 0.2$ & $1.0 \pm 0.2$ & $1.2 \pm 0.2$ & $1.4 \pm 0.4$ & $1.5 \pm 0.5$ & $1.2 \pm 0.5$ & \\
Right buccal OMI & $0.4 \pm 0.1$ & $0.9 \pm 0.2$ & $1.2 \pm 0.2$ & $1.4 \pm 0.4$ & $1.3 \pm 0.5$ & $1.1 \pm 0.5$ & \\
Left lateral tongue OMI & $0.1 \pm 0.1$ & $0.4 \pm 0.2$ & $0.5 \pm 0.2$ & $0.5 \pm 0.2$ & $0.6 \pm 0.3$ & $0.5 \pm 0.3$ & \\
Right lateral tongue OMI & $0.1 \pm 0.1$ & $0.4 \pm 0.2$ & $0.6 \pm 0.2$ & $0.5 \pm 0.2$ & $0.7 \pm 0.3$ & $0.5 \pm 0.3$ & \\
Left buccal xerostomia scale & $-0.5 \pm 0.4$ & $0.8 \pm 0.4$ & $1.1 \pm 0.5$ & $0.3 \pm 0.5$ & $1.0 \pm 0.6$ & $0.0 \pm 0.6$ & \\
Right buccal xerostomia scale & $-0.3 \pm 0.4$ & $1.0 \pm 0.5$ & $1.2 \pm 0.4$ & $0.6 \pm 0.5$ & $1.0 \pm 0.6$ & $0.2 \pm 0.6$ & \\
Left buccal pain scale & $0.6 \pm 0.2$ & $0.7 \pm 0.4$ & $1.6 \pm 0.5^{\mathrm{a}}$ & $0.8 \pm 0.6$ & $1.5 \pm 0.7$ & $0.2 \pm 0.6$ & $<0.05$ \\
Right buccal pain scale & $0.7 \pm 0.3$ & $0.8 \pm 0.4$ & $1.9 \pm 0.5 \mathrm{a}$ & $1.3 \pm 0.5$ & $1.7 \pm 0.6$ & $0.3 \pm 0.6$ & $<0.05$ \\
Throat pain scale & $0.6 \pm 0.3$ & $1.3 \pm 0.6$ & $3.1 \pm 0.6^{\mathrm{a}}$ & $1.8 \pm 0.5$ & $1.4 \pm 0.6$ & $0.6 \pm 0.6$ & \\
\hline
\end{tabular}

${ }^{a}$ Left and right buccal pain scale significant versus throat pain scale at observation 3 .

Data format: average \pm standard error of the mean.

corresponds to a $48 \%$ and $39 \%$ difference in left and right buccal pain, respectively, compared to throat pain.

The incidence and severity of UOM in our study can be found in Figure 3. There was a 53\% incidence of UOM; most of those $(59 \%, 31 \%$ of all patients) were categorized as having mild UOM. Although the study period was shortened to 14 days in order to minimize the influence of GVHD, four patients were diagnosed with the disease within the study period. One patient was categorized as having "severe" UOM, while the other three did not show evidence of UOM.

Interobservervariance analysis revealed a small, though significant $(p<0.05)$ bias toward higher OMI scores for one observer within one observer pair throughout the study. Although this pair made the majority of the observations throughout the study, the average difference between measurements was only 0.08 , and the pair agreed with each other $61 \%$ of the time. Analysis between the other two pairs of observers showed they were not significantly different.

\section{DISCUSSION}

This study represents the first clinical trial of NASAdeveloped LEDs used to treat OM due to myeloablative therapy in pediatric BMT patients. LED treatment produced a significant $48 \%$ and $39 \%$ reduction in treated left and right buccal pain, respectively, compared to the untreated throat. This corresponds to approximately posttransplant day 7 , which is consistent with our preliminary data for this study. ${ }^{18}$

There was also a reduction in the expected incidence of UOM based on historical epidemiological data $(53 \%$ versus 70-90\%). This decrease in the incidence of UOM from expected was noteworthy despite the fact that our measurement for "mild" UOM was very sensitive, requiring only one observer at any time noting ulceration. This coincides with a low incidence of "moderate" (9\%) and "severe" (13\%) UOM in our treated study population.

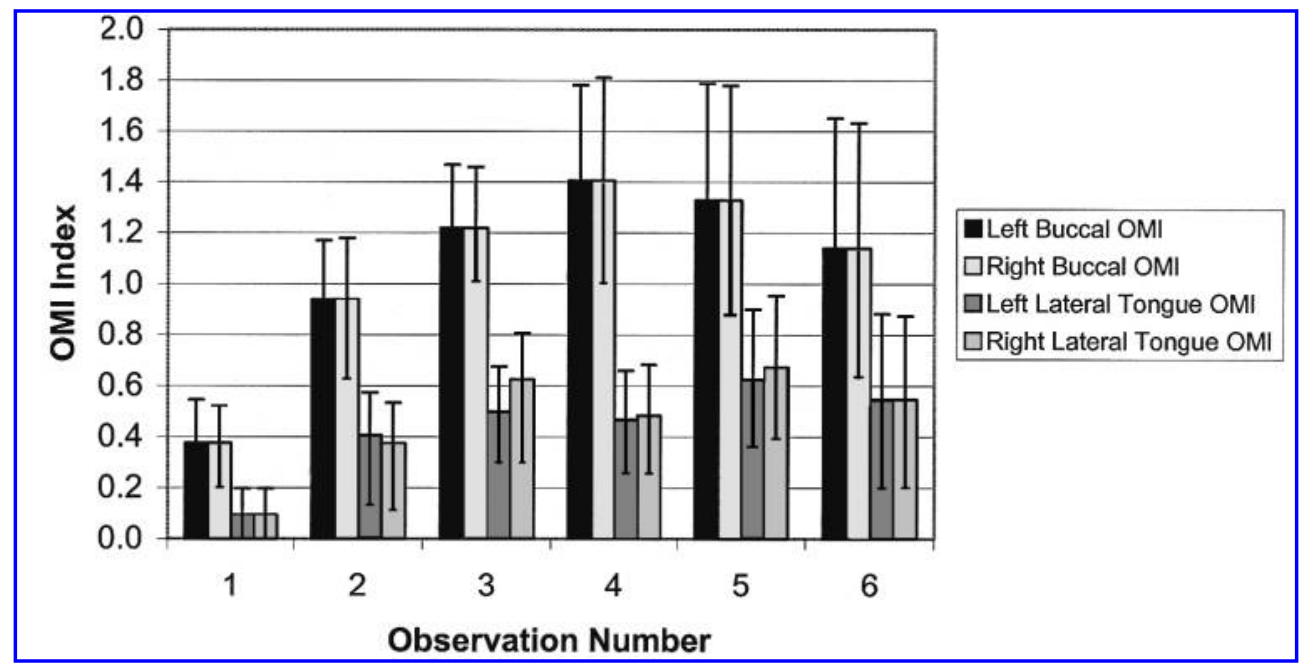

FIG. 1. Average difference from baseline of the OMI of left/right buccal and left/right lateral tongue mucosal surfaces. Error bars represent standard errors of the mean. 


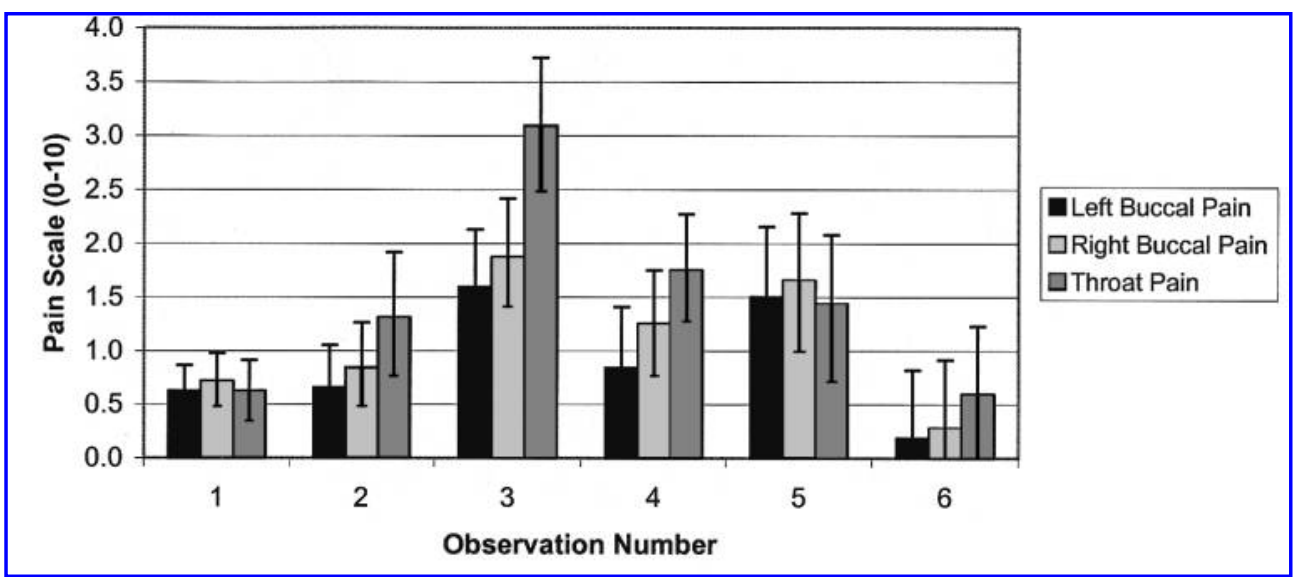

FIG. 2. Change in pain scale from baseline of left and right buccal pain compared to throat pain. Error bars represent standard errors of the mean.

The lack of significance between left and right sides of the buccal OMI, lateral tongue OMI, buccal pain, and buccal xerostomia is also important to note. This is consistent with the deep tissue penetration of near-infrared light, treating both the left as well as the right (where sham treatments occurred) oral mucosa.

Despite the encouraging findings from this study, the protocol design had some disadvantages. Comparison of the OMI of the treated buccal and lateral tongue mucosal surfaces to historical epidemiological controls is difficult. Most studies in the literature reported the entire OMI score as a function of time, not OMI scores of specific sites. Also, the comparison between buccal pain and throat pain may not be as reliable as comparing buccal pain of different sides. Swallowing occurs frequently throughout the day whereas buccal irritation can be controlled more easily, perhaps causing a greater awareness of throat pain. Finally, although the incidence of UOM was reduced from expected rates based on epidemiological data, a randomized and blinded control group within the study would have provided clearer results.

In response to these limitations, we have designed a larger, multicentered, randomized phase 2 , double-blinded trial with patients used as their own concurrent control through the use of an intra-oral shield. This shield will block irradiation of direct and indirect LED light from the opposite side. This will allow for a direct comparison of measured OMI as well as perceived pain and xerostomia of treated and untreated buccal surfaces. We look forward with great anticipation to the results of this trial.

\section{ACKNOWLEDGMENTS}

We wish to thank the clinicians and nursing staff that worked to implement the design study into practice. We also would like to thank Ron Ignatius at Quantum Devices (Barneveld, Wisconsin) for his help in providing the LED arrays used in this study. This work was supported by the Defense Advanced Research Projects Agency (DARPA) grant N66001-01-1-8969, the National Aeronautics and Space Administration (NASA), Marshall Space Flight Center SBIR grants NAS8-99015 and NAS8-97277, the Bleser Endowed Professorship, Children's Hospital Foundation, the Midwest Athletes Against Childhood Cancer (MACC) Fund, and Quantum Devices, Inc.

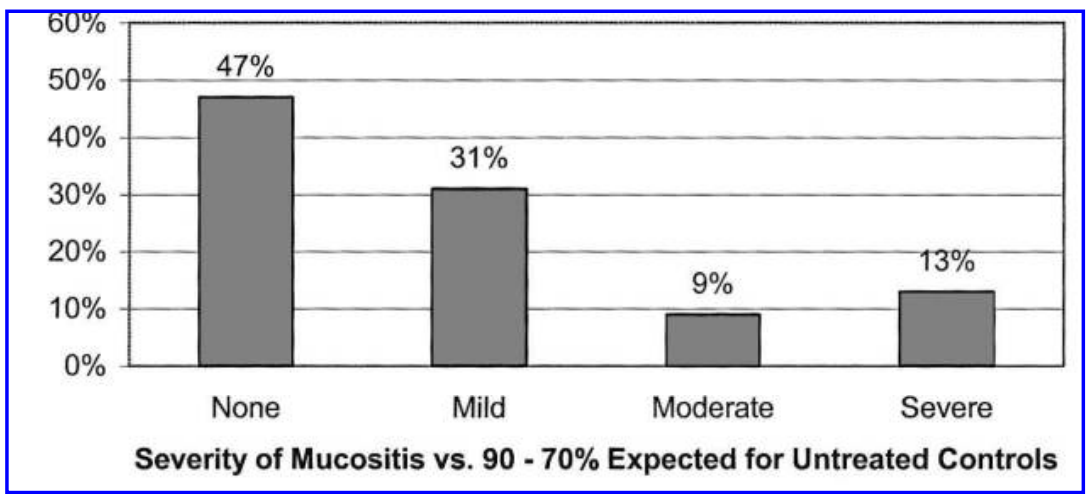

FIG. 3. Incidence of ulcerative oral mucositis in patients treated with LED light. 


\section{REFERENCES}

1. Schubert, M.M., Sullivan, K.M., and Truelove, E.L. (1986). Head and neck complications of bone marrow transplantation. in: Head and neck management of the cancer patient. D.E. Peterson, E.G. Elias, S.T. Sonis (eds.). The Hauge: Martinus Nijhoff, pp. 401-427.

2. Kolbinson, D.A., Schubert, M.M., Flournoy, N., et al. (1988). Early oral changes following bone marrow transplantation. Oral Surg. Oral Med. Oral Pathol. 66, 130-138.

3. Dreizen, S., McCredie, K.B., Dicke, K.A., et al. (1979). Oral complications of bone marrow transplantation: in adults with acute leukemia. Postgrad. Med. 66, 187-194.

4. Beauvoit, B., Kitai, T., and Chance, B. (1994). Correlation between the light scattering and the mitochondrial content of normal tissues and transplantable rodent tumors. Biophysics 67, 2501-2510.

5. Beauvoit, B., Evans, S.M., Jenkins, T.W., et al. (1995). Contribution of the mitochondrial compartment to the optical properties of the rat liver: a theoretical and practical approach. Anal. Biochem. 226, 167-174.

6. Abergel, R.P., Lyons, R.F., Castel, J.C., et al. (1987). Biostimulation of wound healing by lasers: experimental approaches in animal models and in fibroblast cultures. J. Dermatol. Surg. Oncol. 13, 127-133.

7. Yu, W., Naim, J.O., and Lanzafame, R.J. (1994). The effect of laser irradiation on the release of bFGF from 3T3 fibroblasts. Photochem. Photobiol. 59, 167-170.

8. Yu, W., Naim, J.O., and Lanzafame, R.J. (1997). Effects of photostimulation on wound healing in diabetic mice. Lasers Surg. Med. $20,56-63$.

9. Tamura, M. (1993). Non-invasive monitoring of the redox state of cytochrome oxidase in living tissue using near-infrared laser lights. Jpn. Circul. J. 57, 817-824.

10. Mester, E., and Jaszagi-Nagy, E. (1973). The effects of laser radiation on wound healing and collagen synthesis. Studia Biophys. Band. 35, 32-35.

11. Lubart, R., Wollman, Y., Friedman, H., et al. (1992). Effects of visible and near-infrared lasers on cell cultures. J. Photochem. Photobiol. 12, 305-310.

12. Miller, M., and Truhe, T. (1993). Lasers in dentistry: an overview. J. ADA. 124, 32-35.

13. Sommer, A.P., Pinheiro, A.L.B., Mester, A.R., et al. (2001). Biostimulatory windows in low-intensity laser activation: lasers, scanners and NASA's light-emitting diode array system. J. Clin. Laser Med. Surg. 19, 29-34.

14. Karu, T. (1989). Photobiology of low-power laser effects. Health Phys. 56, 691-704.

15. Ciais, G., Namer, M., Schneider, M., et al. (1996). La laserthérapie dans la prevention et le traitement des mucites liées á la chimiothérapie anticancéreuse. Bull. Cancer. 79, 183-191.

16. Barasch, A., Peterson, D.E., Tanzer, J.M., et al. (1995). Helium- neon laser effects on conditioning-induced oral mucositis in bone marrow transplant patients. Cancer 76, 2550-2556.

17. Cowen, D., Tardieu, C., Schubert, M., et al. (1997). Low energy helium-neon laser in the prevention of oral mucositis in patients undergoing bone marrow transplant: results of a double blind randomized trial. Int. J. Radiat. Oncol. Biol. Phys. 38, 697-703.

18. Whelan, H.T., Smits, R.L., Buchmann, E.V., et al. (2001). Effect of NASA light-emitting diode irradiation on wound healing. J. Clin. Laser Med. Surg. 19, 305-314.

19. Whelan, H.T., Buchmann, E.V., Whelan, N.T., et al. (2001). Medial applications of space light-emitting diode technology $\longrightarrow$ space station and beyond. Space Technol. Appl. Int. Forum 458, 3-15.

20. Chance, B., Nioka, S., Kent, J., et al. (1988). Time-resolved spectroscopy of hemoglobin and myoglobin in resting and ischemic muscle. Anal. Biochem. 174, 698-707.

21. Wong-Riley, M.T.T., Xuetao, B., Buchmann, E., et al. (2001). Light-emitting diode treatment reverses the effect of TTX on cytochrome oxidase in neurons. NeuroReport 12, 3033-3037.

22. Whelan, H.T., Houle, J.M., Whelan, N.T., et al. (2000). The NASA light-emitting diode medical program-progress in space flight and terrestrial applications. Space Technol. Appl. Int. Forum 504, $3-15$.

23. Whelan, H.T., Buchmann, E.V., Whelan, N.T., et al. (2001). NASA light-emitting diode medical applications: from deep space to deep sea. Space Technol. Appl. Int. Forum 552, 35-45.

24. Whelan, H.T., Smits, R.L., Buchmann, E.V., et al. (2001). Effect of NASA light-emitting diode irradiation on wound healing. J. Clin. Laser Med. Surg. 19, 305-314.

25. Woo, S.B., Sonis, S.T., Monopoli, M.M., et al. (1993). A longitudinal study of oral ulcerative mucositis in bone marrow transplantation recipients. Cancer 72, 1612-1617.

26. Wingard, J.R., Niehaus, R.D.H., Peterson, D.E., et al. (1991). Oral mucositis after bone marrow transplantation: a marker of treatment toxicity and predictor of hepatic veno-occlusive disease. Oral. Surg. Oral Med. Oral Pathol. 72, 419-424.

27. Barasch, A., Peterson, D.E., Tanzer, J.M., et al. (1995). Heliumneon laser effects on conditioning-induced oral mucositis in bone marrow transplantation patients. Cancer 76, 2550-2556.

28. Schubert, M.M., Williams, B.E., Lloid, M.E., et al. (1992). Clinical assessment scale for the rating of oral mucosal changes associated with bone marrow transplantation. Cancer 69, 2469-2477.

29. Wong, D.L., and Baker, C.M. (1988). Pain in children: comparison of assessment scales. Pediatr. Nurs. 14, 9-17.

Address reprint requests to: Harry T. Whelan, M.D.

Department of Neurology Medical College of Wisconsin Milwaukee, WI 53226

E-mail:hwhelan@mcw.edu 


\section{This article has been cited by:}

1. Brendan J. Quirk, Harry T. Whelan . 2011. Near-Infrared Irradiation Photobiomodulation: The Need for Basic ScienceNear-Infrared Irradiation Photobiomodulation: The Need for Basic Science. Photomedicine and Laser Surgery 29:3, 143-144. [Citation] [Full Text] [PDF] [PDF Plus]

2. Ana Paula Cavalcanti de Sousa, Jean N. Santos, João A. dos Reis , Jr. , Taís A. Ramos , José de Souza , Maria Cristina T. Cangussú, Antônio L.B. Pinheiro . 2010. Effect of LED Phototherapy of Three Distinct Wavelengths on Fibroblasts on Wound Healing: A Histological Study in a Rodent ModelEffect of LED Phototherapy of Three Distinct Wavelengths on Fibroblasts on Wound Healing: A Histological Study in a Rodent Model. Photomedicine and Laser Surgery 28:4, 547-552. [Abstract] [Full Text] [PDF] [PDF Plus]

3. D.H. McDaniel, R.A. Weiss, R.G. Geronemus, C. Mazur, S. Wilson, M.A. Weiss. 2010. Varying ratios of wavelengths in dual wavelength LED photomodulation alters gene expression profiles in human skin fibroblasts. Lasers in Surgery and Medicine 42:6, 540-545. [CrossRef]

4. Motoi Ishiguro, Kazuo Ikeda, Katsuro Tomita. 2010. Effect of near-infrared light-emitting diodes on nerve regeneration. Journal of Orthopaedic Science 15:2, 233-239. [CrossRef]

5. Sang-Gyung Kim, Im-Hee Shin, Chang-Hyuk Choi, Jung-Yoon Choe. 2009. Anti-Inflammatory Effect of Near-Infrared Irradiated Cell Culture Media. The Korean Journal of Laboratory Medicine 29:4, 338. [CrossRef]

6. Leticia Lang-Bicudo, Fernanda De Paula Eduardo , Carlos De Paula Eduardo, Denise Maria Zezell . 2008. LED Phototherapy to Prevent Mucositis: A Case ReportLED Phototherapy to Prevent Mucositis: A Case Report. Photomedicine and Laser Surgery 26:6, 609-613. [Abstract] [PDF] [PDF Plus]

7. Nancy T. Sacono, Carlos A.S. Costa, Vanderlei S. Bagnato, Fabio C.B. Abreu-e-Lima. 2008. Light-emitting diode therapy in chemotherapy-induced mucositis. Lasers in Surgery and Medicine 40:9, 625-633. [CrossRef]

8. Meire Maman Fracher Abramoff, Nilza Nelly Fontana Lopes, Luciana Almeida Lopes, Luciano Lauria Dib , Arnaldo Guilherme, Eliana Monteiro Caran, Adriana Delboni Barreto , Maria Lucia Martinho Lee, Antônio Sérgio Petrilli . 2008. Low-Level Laser Therapy in the Prevention and Treatment of Chemotherapy-Induced Oral Mucositis in Young PatientsLow-Level Laser Therapy in the Prevention and Treatment of Chemotherapy-Induced Oral Mucositis in Young Patients. Photomedicine and Laser Surgery 26:4, 393-400. [Abstract] [PDF] [PDF Plus]

9. Joseph Tafur , Paul J. Mills . 2008. Low-Intensity Light Therapy: Exploring the Role of Redox MechanismsLow-Intensity Light Therapy: Exploring the Role of Redox Mechanisms. Photomedicine and Laser Surgery 26:4, 323-328. [Abstract] [PDF] [PDF Plus]

10. Adalberto Vieira Corazza, Dr. Jacks Jorge, Cristina Kurachi, Vanderlei Salvador Bagnato . 2007. Photobiomodulation on the Angiogenesis of Skin Wounds in Rats Using Different Light SourcesPhotobiomodulation on the Angiogenesis of Skin Wounds in Rats Using Different Light Sources. Photomedicine and Laser Surgery 25:2, 102-106. [Abstract] [PDF] [PDF Plus]

11. Luciane B. Cruz, Anelise S. Ribeiro, Angela Rech, Lauro G.N. Rosa, Cláudio G. Castro, Algemir L. Brunetto. 2007. Influence of low-energy laser in the prevention of oral mucositis in children with cancer receiving chemotherapy. Pediatric Blood \& Cancer 48:4, 435-440. [CrossRef]

12. Seung Yoon Lee, Chung Eui You, Mi Youn Park. 2007. Blue and red light combination LED phototherapy for acne vulgaris in patients with skin phototype IV. Lasers in Surgery and Medicine 39:2, 180-188. [CrossRef]

13. M. Maitland DeLand, Robert A. Weiss, David H. McDaniel, Roy G. Geronemus. 2007. Treatment of radiation-induced dermatitis with light-emitting diode (LED) photomodulation. Lasers in Surgery and Medicine 39:2, 164-168. [CrossRef] 
14. Adalberto Vieira Corazza, Jacks Jorge, Cristina Kurachi, Vanderlei Salvador Bagnato. 2007. Photobiomodulation on the Angiogenesis of Skin Wounds in Rats Using Different Light Sources. Photomedicine and Laser Surgery 25:2, 102. [CrossRef]

15. Chukuka S. Enwemeka . 2006. The Place of Coherence in Light Induced Tissue Repair and Pain ModulationThe Place of Coherence in Light Induced Tissue Repair and Pain Modulation. Photomedicine and Laser Surgery 24:4, 457-457. [Citation] [PDF] [PDF Plus]

16. Dr. Luigi Corti, Vanna Chiarion-Sileni, Savina Aversa, Alberto Ponzoni, Raimondo D'Arcais, Stefano Pagnutti , Davide Fiore, Guido Sotti . 2006. Treatment of Chemotherapy-Induced Oral Mucositis with Light-Emitting DiodeTreatment of Chemotherapy-Induced Oral Mucositis with Light-Emitting Diode. Photomedicine and Laser Surgery 24:2, 207-213. [Abstract] [PDF] [PDF Plus]

17. Kristina D. Desmet, David A. Paz, Jesse J. Corry, Janis T. Eells, Margaret T.T. Wong-Riley, Michele M. Henry, Ellen V. Buchmann, Mary P. Connelly, Julia V. Dovi, Huan Ling Liang, Diane S. Henshel, Ronnie L. Yeager, Deborah S. Millsap, Jinhwan Lim, Lisa J. Gould, Rina Das , Marti Jett , Brian D. Hodgson, David Margolis , Dr. Harry T. Whelan . 2006. Clinical and Experimental Applications of NIR-LED PhotobiomodulationClinical and Experimental Applications of NIR-LED Photobiomodulation. Photomedicine and Laser Surgery 24:2, 121-128. [Abstract] [PDF] [PDF Plus]

18. Shin-ichiro Takezaki, Tokuya Omi, Shigeru Sato, Seiji Kawana. 2006. Light-emitting Diode Phototherapy at $630 \pm 3 \mathrm{~nm}$ Increases Local Levels of Skin-homing T-cells in Human Subjects. Journal of Nippon Medical School 73:2, 75-81. [CrossRef]

19. Robert A. Weiss, David H. McDaniel, Roy G. Geronemus, A. WEISS MARGARET, L. BEASLEY KAREN, Girish M. Munavalli, Supriya G. Bellew. 2005. Clinical Experience with Light-Emitting Diode (LED) Photomodulation. Dermatologic Surgery 31, 1199-1205. [CrossRef]

20. Ronnie L. Yeager, Jill A. Franzosa, Deborah S. Millsap, Jennifer L. Angell-Yeager, Stephen S. Heise , Phoebe Wakhungu, Jinhwan Lim, Harry T. Whelan, Janis T. Eells, Diane S. Henshel . 2005. Effects of 670-nm Phototherapy on DevelopmentEffects of 670-nm Phototherapy on Development. Photomedicine and Laser Surgery 23:3, 268-272. [Abstract] [PDF] [PDF Plus]

21. Robert A. Weiss, David H. McDaniel, Roy G. Geronemus, Margaret A. Weiss. 2005. Clinical trial of a novel non-thermal LED array for reversal of photoaging: Clinical, histologic, and surface profilometric results. Lasers in Surgery and Medicine . [CrossRef]

22. Anne Hendren Coulter . 2003. Let There Be Light - and HealingLet There Be Light - and Healing. Alternative and Complementary Therapies 9:6, 322-326. [Citation] [PDF] [PDF Plus]

23. Helen V Worthington, Jan E Clarkson, Tim OB Eden, Helen V WorthingtonInterventions for preventing oral mucositis for patients with cancer receiving treatment . [CrossRef]

24. Helen V Worthington, Jan E Clarkson, Gemma Bryan, Susan Furness, Anne-Marie Glenny, Anne Littlewood, Martin G McCabe, Stefan Meyer, Tasneem Khalid, Helen V WorthingtonInterventions for preventing oral mucositis for patients with cancer receiving treatment . [CrossRef] 